\title{
La percepción de los estilos de crianza y su relación con las autopercepciones de los niños de Buenos Aires: Diferencias en función del género*
}

Perceived Parenting Styles and their Relation to Self-perceptions of Children from Buenos Aires: Differences by Gender

\begin{abstract}
María Fernanda Molina ${ }^{a}$
Universidad de Buenos Aires, Argentina

Consejo Nacional de Investigaciones Científicas y

Técnicas (CONICET), Argentina

ORCID: http://orcid.org/0000-0002-7361-7058

María Julia Raimundi

Universidad de Buenos Aires, Argentina

Consejo Nacional de Investigaciones Científicas y

Técnicas (CONICET), Argentina

Lucía Bugallo

Instituto Patagónico de Estudios de Historia y Ciencias

Sociales (IPEHCS), Argentina

Consejo Nacional de Investigaciones Científicas y Técnicas
\end{abstract}

(CONICET), Argentina

a Autor de correspondencia. Correo electrónico: fer_molina@hotmail.com

Para citar este artículo: Molina, M.F., Raimundi, M.J., \& Bugallo, L. (2017). La percepción de los estilos de crianza y su relación con las autopercepciones de los niños de Buenos Aires: Diferencias en función del género. Universitas Psychologica 16(1), 1-12. http://dx. doi.org/10.11144/Javeriana.upsy16-1.pecr
Recepción: 05 Febrero 2014 | Aprobación: 23 Enero 2017

\section{RESUMEN}

El objetivo de este trabajo fue estudiar la influencia de la percepción de los estilos de crianza en las autopercepciones de los niños en función del género. Se administró el Perfil de Autopercepciones para Niños (SPPC) y la Escala Argentina de Percepción de la Relación con los Padres a una muestra de 98 niños de ambos sexos (varones $43.90 \%$; edad media $=9.86 ; D E=1.28$ ) de Buenos Aires (Argentina). Se encontró que la percepción de la relación con los padres predice el nivel de las autopercepciones de las niñas en los todos los dominios evaluados (físico/social, académico/comportamental, y autoestima global) mientras que solo la aceptación por parte de la madre predice las autopercepciones de los varones en el dominio físico/social..

Palabras clave

estilos de crianza; relación padres-hijos; autoconcepto; autoestima; niños; percepción.

\footnotetext{
ABSTRACT

The aim of this work was to study the influence of perceived parenting styles in children's self-perceptions considering differences by gender. The Self-Perception Profile for Children (SPPC) and the Argentine Scale of the Perception of the Relationship with Parents was administered to a sample of 98 children of both genders (males $43.90 \%$, mean $=9.86$ years, $S D=$ 1.28) of Buenos Aires (Argentina). We found that the perception of the relationships with parents predicted the level of girls' self-perceptions in all the assessed domains (physical/social, academic/behavioral, and global self-esteem) while only the acceptance perceived in the relationship with the mother predicted boys' self-perceptions in the physical/social domain. Keywords parenting styles; parent-child relationship; self-concept; self-esteem; children; perception.
} 
Desde que nacen, las personas se desarrollan en el seno familiar. En él crecen, constituyen su identidad, adquieren responsabilidades y compromisos, y desarrollan recursos que les permiten ingresar en el mundo adulto (Minichiello, Maglio, \& Schmidt, 2010).

Entre estos recursos, el autoconcepto y la autoestima cumplen una función fundamental para un desarrollo infanto-juvenil saludable. Una autopercepción positiva se asocia a un mayor bienestar subjetivo (RodríguezFernández, Ramos-Díaz, Ros, Fernández-Zabala, \& Revuelta, 2016), una mayor resiliencia (Cardozo \& Alderete, 2009), un desempeño académico favorable (e.g. Jiang, Song, Lee, \& Bong, 2014; Miñano, Gilar, \& Castejón, 2012), una actitud positiva hacia la escuela y el estudio (Schmidt, Messoulam, \& Molina, 2008), una mayor implicación escolar (RamosDíaz, Rodríguez-Fernández, Fernández-Zabala, Revuelta, \& Zuazagoitia, 2016), una menor predisposición a la depresión (e.g. Jiménez, Murgui, \& Musitu, 2007; Wu \& Kuo, 2014) e ideación suicida (de la Torre, Cubillas Rodríguez, Román Pérez, \& Valdez, 2009), menores niveles de comportamiento agresivo (Çiftçi, 2015), y una menor ansiedad e inadaptación (Acevedo Ponce de León \& Carrillo Árcega, 2010). Esto muestra la importancia de indagar los factores que pueden intervenir en el desarrollo de una visión positiva del sí mismo.

Las autopercepciones se constituyen a partir de la interacción de múltiples factores. Entre ellos, las interacciones tempranas en el entorno familiar y con los padres en particular tienen un peso fundamental (Harter \& Bukowski, 2012). Son dichas interacciones las que permiten al niño ganar entendimiento sobre sí mismo, tanto subjetiva como objetivamente (Coplan, Findlay, $\&$ Nelson, 2004).

Particularmente, los estilos de crianza son un elemento clave en la constitución del autoconcepto de los hijos (Harter, 1999; Muñoz Silva, 2005; Torío López, Peña Calvo, \& Rodríguez Menéndez, 2008; Vargas Rubilar \& Oros, 2013) y es la percepción que tienen los niños sobre las conductas de sus padres la que tiene la mayor influencia (e.g. Ivanova \& Israel, 2006; Rodríguez, del Barrio, \& Carrasco, 2009).

En este sentido, la percepción de aceptación, calidez, y afecto en la relación con los padres se asocia al desarrollo de autopercepciones más positivas en los niños. A su vez, las formas positivas de control (i.e. el control conductual, la provisión de estructura) y la promoción de autonomía se asocian con una autoestima más elevada y un autoconcepto más positivo, mientras que las formas negativas de control (i.e. el control psicológico, la coerción, el caos) se asocian con autopercepciones más negativas (e.g. Clerici \& García, 2011; Frank, Plunkett, \& Otten, 2010; Fuentes, García, Gracia, \& Alarcón, 2014; Reina, Oliva Delgado, \& Parra Jiménez, 2010; Richaud de Minzi, 2006a; Siffert, Schwarz, \& Stutz, 2012).

Esto demuestra la relevancia de estudiar cómo influye la forma en que los niños perciben los estilos de crianza de sus padres en sus autopercepciones. Ahora bien, iinfluyen de la misma manera el padre y la madre en las autopercepciones de sus hijos?

Las investigaciones sobre los patrones parentales de crianza, se han focalizado principalmente en la madre, dejando de lado la influencia del padre (De La Iglesia, Ongarato, \& Fernández Liporace, 2011). Sin embargo, existen evidencias de que tanto las madres como los padres tienen una gran importancia psicológica como figuras apego y, de que ambos desempeñan roles diferentes en lo que respecta a las funciones parentales (Lamb \& Tamis-Lemonda, 2004).

Por otro lado, las diferencias de género en los hijos también deben ser tenidas en cuenta ya que existen diferencias en la valencia y en el contenido de las autopercepciones en función del sexo (Harter, 1999). Los varones tienden a presentar autopercepciones más positivas que las niñas. No obstante, en el dominio del comportamiento las niñas se perciben más competentes que los varones (e.g. Molina, Raimundi, López, Cataldi, \& Bugallo, 2011; Gorostegui, \& Dörr, 2005).

Esto plantea el interrogante sobre si la percepción de la relación con el padre y con 
la madre tiene un efecto diferente en las autopercepciones de los niños y las niñas.

El objetivo de este estudio fue explorar cómo influye la percepción del estilo de crianza del padre y la madre en las autopercepciones de los niños y niñas del Conurbano Bonaerense (Provincia de Buenos Aires, Argentina). Se adoptó el enfoque dimensional y se indagaron las dos principales dimensiones de los estilos parentales: la afectiva y la del control.

Hipótesis

1. la percepción de aceptación por parte de los padres predice autopercepciones más positivas en los hijos.

2. la percepción de formas moderadas de control predice autopercepciones más positivas.

3. la percepción de formas extremas de control predice autopercepciones más negativas.

4. la percepción de falta de control predice autopercepciones más negativas.

Por último, se estudió si el género de los padres y los hijos ejerce un efecto moderador en la relación entre los estilos parentales percibidos y las autopercepciones de los niños. Este objetivo fue exploratorio y por lo tanto no se plantearon hipótesis al respecto.

\section{Método}

Tipo de estudio

Se realizó un estudio descriptivo correlacional con un diseño no experimental de tipo transaccional (Hernández Sampieri, FernándezCollado, \& Baptista Lucio, 2010).

\section{Participantes}

Se incluyeron niños escolarizados de ambos sexos, de entre 8 y 12 años edad. La muestra utilizada fue de tipo no probabilística o dirigida por conveniencia, de sujetos voluntarios (Hernández Sampieri et al., 2010). Estuvo integrada por niños $(n=98)$ de ambos sexos (56.1\% de mujeres) de escuelas de enseñanza primaria del Conurbano Bonaerense con una edad promedio de $9.86(D E=1.28)$. La mayoría de los padres estaban casados o conviviendo (78.70\%) y tenían un nivel de instrucción secundario completo o superior (madres $=84.40$ $\%$; padres $=91.60 \%$ ). La mayoría de los niños pertenecía a un hogar biparental (74.07\%).

\section{Procedimientos}

Los niños participaron de forma voluntaria, anónima y brindaron su consentimiento informado oralmente. Sus padres previamente firmaron un consentimiento escrito. Las escalas fueron administradas en forma grupal por evaluadores entrenados. Las consignas fueron leídas en voz alta y explicadas por los evaluadores, luego los cuestionarios fueron respondidos en forma individual.

\section{Instrumentos}

\section{Cuestionario sociodemográfico}

Fue construido ad-hoc para caracterizar la muestra en función de sus características sociodemográficas (edad, sexo, nivel educativo de los padres, estado civil y composición familiar).

Perfil de Autopercepciones para Niños (SPPC, Harter, 1985; Adaptación: Molina et al., 2011)

Posee 36 ítems y evalúa el autoconcepto a través de cinco dominios específicos: competencia académica, aceptación social, comportamiento, competencia deportiva y apariencia física. Por otra parte, evalúa la autoestima global con una subescala especialmente diseñada para tal fin. Esta variable es definida como el grado en que el niño se agrada como persona y está feliz con la forma en que está conduciendo su vida.

Cada subescala se compone de seis ítems con cuatro opciones de respuesta. El formato de respuesta consiste en dos frases contrapuestas 
que muestran dos grupos de niños con autopercepciones opuestas. Los niños deben elegir en primer lugar, a qué grupo se parecen y, en segundo lugar, en qué grado se parecen a ese grupo (Realmente como yo o Más o menos como yo). Los ítems son del tipo: "Algunos chicos pueden aprender con facilidad lo que se les enseña en la escuela Pero Otros chicos tienen dificultad para aprender lo que se les enseña" (competencias académicas) o "Algunos chicos, a menudo, no están contentos con ellos mismos Pero Otros chicos están bastante satisfechos con ellos mismos" (autoestima global).

En su versión original el SPPC ha mostrado tener una adecuada validez factorial y buena consistencia interna (alpha de Cronbach de 0.71 a 0.86) (Harter, 1985). En la versión local de la escala se encontraron evidencias a favor de la validez de constructo, factorial, convergente y discriminante. En cuanto a la confiabilidad de la escala, los índices de consistencia interna son de adecuados a buenos (alpha de Cronbach de 0.71 a 0.84) (Molina et al., 2011).

Las autopercepciones de dominio específico pueden agruparse en dos dimensiones más generales: el dominio físico/social y el académico/ comportamental (Harter, 1999). En este trabajo se estudiaron estos dominios de la autopercepción y la autoestima global. El puntaje para las autopercepciones en el dominio físico/ social se obtuvo a partir de la sumatoria de las puntuaciones en los dominios de la aceptación social, las competencias deportivas y la apariencia física. El puntaje para las autopercepciones en el dominio académico/comportamental se obtuvo por medio de la sumatoria de las puntuaciones en las subescalas competencias académicas y comportamiento. Las tres subescalas mostraron una consistencia interna (alpha de Cronbach) adecuada $(\alpha=0.70)$.

Escala Argentina de percepción de la relación con los padres para niños de 8 a 12 años (EAPRP, Richaud de Minzi, 2007)

Evalúa la percepción que tiene el niño de la aceptación por parte de su padre y su madre y tres aspectos del control en la relación con ellos. La escala está compuesta por 32 ítems con tres opciones de respuestas (siempre, a veces, nunca) que se dividen en cuatro subescalas: aceptación, control aceptado, control patológico y autonomía extrema. Los ítems son del tipo "Mi mamá me dice que me quiere mucho" (aceptación), "Mi mamá controla que llegue a casa a horario" (control aceptado), "Mi mamá cree que castigándome va a corregir mi mal comportamiento" (control patológico), "Mi mamá me deja hacer lo que quiera" (autonomía extrema). Las versiones de la madre y el padre están compuestas por los mismos ítems.

La escala presenta una adecuada validez de contenido, validez factorial y según criterio. Los índices de consistencia interna (alpha de Cronbach) son de adecuados a muy buenos (de 0.60 a 0.92 para la madre y de 0.60 a 0.89 para el padre) (Richaud de Minzi, 2007).

En este estudio los coeficientes de consistencia interna obtenidos fueron de regulares a buenos (aceptación, 0.68 para la madre y 0.74 para el padre; control aceptado, 0.51 para la madre y 0.58 para el padre; control patológico, 0.69 para la madre y 0.69 para el padre; autonomía extrema, 0.68 para la madre y 0.75 para el padre).

\section{Análisis de datos}

Se utilizaron correlaciones ( $r$ de Pearson) para estudiar la asociación entre la percepción de la relación con los padres y las autopercepciones de los niños. El tamaño del efecto fue estudiado con el coeficiente $r$ de Pearson, siguiendo los criterios de Cohen (1992).

Se realizaron análisis de regresión lineal simple y múltiple por el método estándar. Se probaron distintos modelos con las variables de la percepción de la relación con los padres (variables independientes) que mostraron tener una relación significativa o marginal con las autopercepciones en los dominios estudiados (variables dependientes). Se realizaron análisis separados en función del género de los padres y los niños. Para cada uno de los modelos, se 
calculó el tamaño del efecto con el estadístico $f^{2}$ de Cohen (1992).

\section{Resultados}

\section{Análisis preliminares}

Al analizar la relación con la madre, en el caso de las niñas, se encontró una asociación positiva entre la percepción de aceptación y las autopercepciones en el dominio académico/ comportamental $(r=0.34, p=0.012)$ y la autoestima global $(r=0.34, p=$ 0.011). En el dominio físico/social, una mayor percepción de autonomía extrema se asoció a una autopercepción más positiva $(\mathrm{r}=$ 0.46, p < 0.001). La percepción de control patológico correlacionó de manera negativa con la autoestima global $(r=-0.31, p=0.022)$. En el caso de los niños, solo se observó una correlación positiva significativa entre la aceptación y las autopercepciones en el dominio físico/social $(r=$ $0.31, p=0.040$ ). En todos los casos, el tamaño del efecto fue mediano.

En cuanto a la percepción de la relación con el padre, en el grupo de las niñas, el control patológico se asoció de manera negativa con las autopercepciones en el dominio físico/social $(r$ $=-0.31, p=0.027)$ y de la autoestima global $(r=-0.36, p=0.007)$. No se encontraron correlaciones significativas en el grupo de los varones.

\section{Análisis principal}

En primer lugar, se analizó la adecuación de los modelos estudiados. A partir del análisis del gráfico de distribución de los residuos se comprobó la normalidad en dicha distribución. Por medio del análisis de los gráficos de probabilidad normal de residuos y de dispersión de los residuos se corroboró el cumplimiento del supuesto de homosedasticidad para todos los modelos (Chica Olmo \& Frías Jamilena, 2000; Ferrán Aranaz, 1996). Por otra parte, los valores obtenidos por el Test de Durbin-Watson (de 1.66 a 2.48) son adecuados, lo que indica la ausencia de autocorrelación de los residuos (Pardo \& Ruiz, 2005). Por último, en el caso de los modelos con más de una variable independiente, se obtuvieron coeficientes de tolerancia adecuados $(0.95$ a 0.99) indicando la ausencia de multicolinealidad (Hair, Anderson, Tatham, \& Black, 2001).

En la Tabla 1 se presentan los resultados de los modelos de la percepción de la relación con la madre que mostraron predecir de manera significativa el autoconcepto y la autoestima global de las niñas. El principal predictor de las autopercepciones en el dominio físico/social fue la autonomía extrema. El porcentaje de varianza explicada por el modelo fue del $20 \%$. El tamaño del efecto fue mediano. El principal predictor de las autopercepciones en el dominio académico/ comportamental fue la aceptación. El porcentaje de varianza explicada por el modelo fue del 10 \%. El tamaño de efecto fue bajo. Por último, los principales predictores de la autoestima global fueron la aceptación y el control patológico. El porcentaje de varianza explicada por el modelo fue del $17 \%$. El tamaño de efecto del modelo fue mediano.

\section{TABLA 1}

Modelos de regresión lineal de las dimensiones de la relación con la madre que predicen las autopercepciones de las niñas

\begin{tabular}{|c|c|c|c|c|c|c|c|}
\hline \multirow[b]{2}{*}{ Predictores } & \multicolumn{3}{|c|}{ Datos de los predictores } & \multicolumn{4}{|c|}{ Datos del modelo } \\
\hline & $B$ & $\beta$ & $95 \%$ IC $B$ & $R^{2}$ & $R^{2}$ ajustado & $F$ & $f^{2}$ \\
\hline \multicolumn{8}{|c|}{ Autoconcepto físico/social } \\
\hline Autonomía extrema & 2.33 & $0.46 * * *$ & {$[1.09,3.58]$} & 0.21 & 0.20 & $14.08^{* * *}$ & 0.26 \\
\hline \multicolumn{8}{|c|}{ Autoconcepto académico/comportamental } \\
\hline Aceptación & 1.07 & $0.34 *$ & {$[0.25,1.89]$} & 0.11 & 0.10 & $6.81^{*}$ & 0.12 \\
\hline \multicolumn{8}{|c|}{ Autoestima global } \\
\hline Aceptación & 0.07 & $0.32 *$ & {$[0.02,0.12]$} & 0.20 & 0.17 & $6.47^{* *}$ & 0.25 \\
\hline Control patológico & -0.05 & $-0.29^{*}$ & {$[-0.09,-0.01]$} & & & & \\
\hline
\end{tabular}

En la Tabla 2 se presentan los resultados del modelo de la percepción de la relación con la madre, que predijo de manera significativa el autoconcepto en el dominio físico/social de los niños. El principal predictor fue la percepción de aceptación por parte de la madre. El porcentaje de varianza explicada por el modelo fue del $8 \%$. El tamaño de efecto fue bajo. 


\section{TABLA 2}

Modelos de regresión lineal de las dimensiones de la relación con la madre que predicen las autopercepciones en el dominio físico/social para los niños

\begin{tabular}{|c|c|c|c|c|c|c|c|}
\hline \multirow[b]{2}{*}{ Predictores } & \multicolumn{3}{|c|}{ Datos de los predictores } & \multicolumn{4}{|c|}{ Datos del modelo } \\
\hline & $B$ & $\mathrm{~B}$ & $\begin{array}{c}95 \% \text { IC para } \\
B\end{array}$ & $R^{2}$ & $R^{2}$ ajustado & $F$ & $f^{2}$ \\
\hline Aceptación & 1.36 & $0.31^{*}$ & {$[0.06,2.66]$} & 0.10 & 0.08 & $4.49^{*}$ & 0.11 \\
\hline
\end{tabular}

Nota: IC $=$ Intervalo de Confianza. ${ }^{*} p<0.05$. Fuente: elaboración propia.

En la Tabla 3, se presentan los resultados de los modelos de la percepción de la relación con el padre, que predijeron significativamente las autopercepciones de las niñas. En el dominio físico/social, una elevada aceptación y un bajo control patológico fueron predictores de una autopercepción más positiva. El porcentaje de varianza explicada por el modelo fue del $18 \%$. El tamaño de efecto fue mediano. Estas mismas variables predijeron la autoestima global de las niñas. El porcentaje de varianza explicada por el modelo fue del $24 \%$. El tamaño del efecto fue alto.

\section{TABLA 3}

Modelos de regresión lineal de las dimensiones de la relación con el padre que predicen las autopercepciones de las niñas

\begin{tabular}{|c|c|c|c|c|c|c|c|}
\hline \multirow[b]{2}{*}{ Predictores } & \multicolumn{3}{|c|}{ Datos de los predictores } & \multicolumn{4}{|c|}{ Datos del modelo } \\
\hline & $B$ & B & $95 \%$ IC $B$ & $R^{2}$ & $\begin{array}{c}R^{2} \\
\text { ajustado }\end{array}$ & $F$ & $f^{2}$ \\
\hline \multicolumn{8}{|c|}{ Autoconcepto físico/social } \\
\hline Aceptación & 1.40 & $0.33^{*}$ & {$[0.30,2.51]$} & 0.21 & 0.18 & $6.39^{* *}$ & 0.26 \\
\hline $\begin{array}{l}\text { Control } \\
\text { patológico }\end{array}$ & -1.14 & $-0.40^{* *}$ & {$[-1.90,-0.39]$} & & & & \\
\hline \multicolumn{8}{|c|}{ Autoestima global } \\
\hline Aceptación & 0.55 & $0.39 * *$ & {$[0.19,0.90]$} & 0.27 & 0.24 & $9.20^{* * *}$ & 0.37 \\
\hline $\begin{array}{l}\text { Control } \\
\text { patológico }\end{array}$ & -0.43 & $-0.45^{* * *}$ & {$[-0.68,-0.19]$} & & & & \\
\hline
\end{tabular}

Nota: IC $=$ Intervalo de Confianza.

$* p<0.05 ; * * p<0.01 ; * * p<0.001$.

Fuente: elaboración propia.

Con respecto a la percepción de la relación con el padre, no se encontraron modelos explicativos para los varones.

\section{Discusión y conclusiones}

Este trabajo tuvo como objetivo explorar cómo influye la aceptación y el control percibidos en la relación con el padre y con la madre en las autopercepciones de los niños y niñas del Conurbano Bonaerense (Provincia de Buenos Aires, Argentina).

A partir de los análisis realizados, se encontró que las dimensiones de los estilos parentales, tal como son percibidas por los niños, predicen de manera diferente el nivel de sus autopercepciones cuando se considera el género de los padres y los hijos.

En el caso de las niñas, la percepción de la relación con la madre impacta en todos los dominios de las autopercepciones estudiados. Por un lado, la percepción de altos niveles de autonomía extrema predice un autoconcepto más positivo en el dominio físico/social. Por otro, la percepción de una mayor aceptación predice un autoconcepto más positivo en el dominio académico/comportamental. Por último, la percepción de altos niveles de aceptación y bajos niveles de control patológico predicen una elevada autoestima global. Esta misma combinación de variables, percibida en la relación con el padre, predijo el nivel del autoconcepto en el dominio físico/social y de la autoestima global de las niñas.

En el caso de los niños, solo la percepción de aceptación en la relación con la madre mostró tener poder predictivo en el dominio físico/social del autoconcepto. La percepción de la relación con el padre no tuvo poder predictivo, para los varones, en ninguno de los dominios evaluados.

Estos resultados presentan evidencias a favor de la primera y la tercera hipótesis: la percepción de aceptación por parte de los padres se asocia a autopercepciones más positivas mientras que el control patológico predice autopercepciones más negativas. Dichos hallazgos son coherentes con los presentados por otros estudios que muestran que la percepción de aceptación, calidez o afecto en la relación con los padres se vinculan con autopercepciones más positivas. Por el contrario, las formas negativas de control (el control psicológico, la coerción y la intrusividad) se asocian a autopercepciones más negativas (Frank et al., 2010; Reina et al., 2010; Siffert et al., 2012).

Por otro lado, no se encontraron evidencias a favor de la segunda hipótesis. La percepción 
de control aceptado no mostró tener poder predictivo para ninguno de los dominios estudiados. Este hallazgo contradice lo indicado por los estudios que muestran que las formas positivas de control, como el control conductual, la provisión de estructura o el control firme se asocian con autopercepciones más positivas (Alonso García \& Román Sánchez, 2005; Oliva Delgado, Parra Jiménez, \& Arranz, 2008; Reina et al., 2010). Sin embargo, en un estudio realizado con niños de nuestro contexto tampoco se encontró relación entre esta dimensión y las autopercepciones de los niños (Richaud de Minzi, 2006a).

La cuarta hipótesis planteada no contó con evidencias que la respalden. No obstante, la percepción de autonomía extrema tuvo poder predictivo del nivel de las autopercepciones pero en el sentido contrario al hipotetizado; predijo autopercepciones más positivas en el dominio físico/social. La autonomía extrema es definida como disciplina laxa y desinterés (Richaud de Minzi, 2007). En nuestro contexto se ha asociado a un funcionamiento negativo en los niños. Altos niveles de autonomía extrema se asocian a mayores conflictos en las relaciones de amistad (Richaud de Minzi, 2006b), afinidad por la soledad y una autopercepción de competencias académicas más negativa (Richaud de Minzi, 2006a).

De todos modos, es importante tener en cuenta que, desde la perspectiva de la tipología de los estilos parentales, las dimensiones de aceptación y control, se combinan derivando en diferentes tipos de estilos de crianza, según con qué otros comportamientos parentales se asocie, la autonomía va a tener diferentes consecuencias en la crianza de los hijos. Es decir, si es acompañada por la falta de compromiso con la crianza va a ser propia de un estilo parental negligente. Sin embargo, también puede ser característica de un estilo de crianza permisivo, en el que la exigencia de los padres es baja pero hay un alto compromiso por parte de ellos y una elevada aceptación (Muñoz Silva, 2005; Torío López et al., 2008). Existen hallazgos que muestran que un estilo parental permisivo es beneficioso (en ocasiones aún más que el autoritativo) para el autoconcepto de los jóvenes de países latinoamericanos (e.g. Brasil en Martínez, García, \& Yubero, 2007; México en Villalobos, Cruz, \& Sánchez, 2004). Por lo tanto, futuras investigaciones deberán profundizar en esta cuestión indagando el rol que juega la autonomía extrema en combinación con otras características de la relación con los padres (e.g. aceptación).

Ahora bien, los resultados encontrados en este estudio difirieron en función del sexo de los padres y de los hijos, lo cual indica que el género, tanto de los hijos como de los padres, cumple un rol moderador en la relación entre la percepción de las pautas de crianza ejercidas por los padres y las autopercepciones de los niños.

Por una parte, la percepción de la relación con los padres tiene mayor influencia para las niñas que para los niños. Estos resultados coinciden con los encontrados en un estudio realizado con niños de Entre Ríos (Argentina). En este trabajo se encontró que las emociones positivas maternas predicen el autoconcepto de las niñas en mayor medida que el de los niños (Resett, Meier, García Sánchez, \& Katz, 2016). Estos hallazgos pueden vincularse con las diferencias que existen en la forma en que los hombres y las mujeres se perciben a ellos mismos y su mundo. Las mujeres son más proclives a tener autoesquemas colectivistas, ensamblados o conectados. En estos esquemas las relaciones con los otros, en especial los otros significativos, son elementos cruciales del self. Los hombres son más propensos a tener autoesquemas individualistas, independientes o autónomos. Es decir, los otros son representados como distintos del self (Cross \& Madson, 1997; Josephs, Markus, \& Romin, 1992).

En tanto la relación con los demás es un aspecto importante en la vida psicológica de las mujeres, es esperable que su autoestima se base, en mayor medida que la de los hombres, en sostener relaciones significativas con otros (Josephs et al., 1992; Oyserman \& Fryberg, 2006). Esto es coherente con lo encontrado en un estudio en el que se indagó cuáles son los principales dominios del autoconcepto que predicen la autoestima de los niños y niñas de nuestro contexto. Para las niñas, los 
principales predictores de la autoestima global fueron la autopercepción de aceptación social y comportamiento, mientras que para los niños el principal predictor de la autoestima fue la autopercepción de apariencia física. Esto muestra la relevancia que toman las dimensiones sociales por sobre las individuales para las niñas de nuestra cultura (Molina \& Raimundi, 2011). Es decir, la aceptación social implica la percepción de aceptación por parte de los pares y la de comportamiento está asociada al comportamiento valorado por los adultos. En cambio, la apariencia física, que tiene la mayor importancia relativa para los varones, constituye la cara externa del self y uno de sus aspectos más personales (Harter, 1999).

En síntesis, es posible que los padres tengan más impacto en las autopercepciones de las niñas porque estas están más abiertas a la influencia del contexto que los varones y, por lo tanto, su autoconcepto está más modelado por las relaciones con sus otros significativos.

Por otra parte, la percepción de la relación con la madre es la que tiene la mayor influencia para ambos sexos. Distintos autores coinciden en que aún hoy las madres pasan más tiempo con sus hijos que los padres y tienen mayores responsabilidades vinculadas con la crianza (Cabrera, Fagan, \& Schadler, 2012; Lamb \& Tamis-Lemonda, 2004; Rodríguez et al., 2009). Es posible que por esta razón sus conductas tengan un mayor impacto en el desarrollo de las autopercepciones de los hijos que las de los padres.

Este estudio presenta algunas limitaciones. Por un lado, se trabajó con una muestra no probabilística y, por lo tanto, no representativa. Es por esto que los resultados deberían ser replicados con otras muestras. Asimismo, existen limitaciones geográficas, los niños que participaron del estudio eran residentes del Conurbano Bonaerense. Por esta razón, las conclusiones alcanzadas en este trabajo no pueden generalizarse a otros contextos de la República Argentina.

Por otro lado, el bajo número de casos no permitió combinar las dimensiones estudiadas de la relación con los padres de forma de poder establecer cómo influye el tipo de estilo parental percibido por el niño en sus autopercepciones. En futuros estudios, se recomienda complementar el enfoque dimensional adoptado en este trabajo con el enfoque tipológico propuesto por Baumrind (1991).

Por último, es importante considerar que las relaciones encontradas, en su mayoría, tienen una magnitud (tamaño de efecto) de mediana a baja. Es decir, que existen otras variables involucradas en la predicción de las autopercepciones infantiles. En este sentido es necesario señalar la complejidad que tiene el origen de la percepción del sí mismo y la multiplicidad de factores involucrados en su constitución. Por un lado, además de los factores sociales, existen otros factores determinantes en su conformación como, por ejemplo, los cognitivos (Harter, 1999). Por el otro, los factores sociales involucran más que la relación con los padres. La relación con los pares y docentes es particularmente relevante para la etapa vital que fue considerada en este estudio (ver Swann \& Bosson, 2010 para una revisión). Es importante indagar estas variables sobre todo en el caso de los varones, ya que la percepción de la relación con los padres ha mostrado tener escasa influencia en sus autopercepciones.

Más allá de estas limitaciones, este estudio realiza aportes tanto teóricos como prácticos. Por un lado, permite conocer en mayor profundidad cómo se vinculan la aceptación y control percibidos en la relación con el padre y la madre con las autopercepciones de los hijos y las hijas. De esta forma, se amplía el conocimiento existente sobre uno de los principales antecedentes del autoconcepto y la autoestima: la relación con las personas que desempeñan un papel central en la crianza.

Por otro lado, el conocimiento de los factores involucrados en el origen de las autopercepciones posibilita desarrollar intervenciones más adecuadas para mejorar la visión que los niños tienen de ellos mismos. Existen evidencias de que los tratamientos destinados a aumentar la autoestima mejoran los puntajes en tests estandarizados de rendimiento, reducen los reportes disciplinarios en la escuela, 
y el uso de drogas y alcohol (ver Swann\& Bosson, 2007 para una revisión). Por esta razón desarrollar programas de intervención para mejorar las autopercepciones ha sido un tema de creciente interés (Bos, Muris, Mulkens, \& Schaalma, 2006; Mann, Hosman, Schaalman, $\&$ de Vries, 2004). Los hallazgos de este estudio realizan un aporte en este sentido, en tanto ponen en evidencia la importancia de la percepción de la relación con ambos padres para las autopercepciones de las niñas. Asimismo, plantea la importancia de la relación con la madre para las autopercepciones de los varones en el dominio físico/social y de considerar otros factores involucrados en el origen de las autopercepciones cuando se trabaja con ellos.

\section{Agradecimientos}

Esta investigación fue financiada por el Consejo Nacional de Investigaciones Científicas y Técnicas (CONICET; Res. No 3609/11 Director: Dra. Schmidt. Co-Director: Dra. Leibovich de Figueroa) y por la Secretaria de Ciencia y Técnica de la Universidad de Buenos Aires (UBACyT 20020100100052; Director: Dra. Leibovich de Figueroa. Co-Director: Dra. Schmidt). Se agradece a los alumnos, docentes, y autoridades del Colegio Alas-Los Pinitos su colaboración en este proyecto de investigación. Asimismo, se agradece a las Lic. Mariel Giménez y Yanina Guzmán su colaboración en la recolección de datos para esta investigación.

\section{Referencias}

Acevedo Ponce de León, J. A., \& Carrillo Árcega, M. L. (2010). Adaptación, ansiedad y autoestima en niños de 9 a 12 años: una comparación entre escuela tradicional y montessori. Psicología Iberoamericana, 18(1), 19-29. Recuperado de http://www.r edalyc.org/articulo.oa?id=133915936003

Alonso García, J., \& Román Sánchez, J. M. (2005). Prácticas educativas familiares y autoestima. Psicothema, 17(1), 76-82.
Recuperado de http://dialnet.unirioja.es/ser vlet/articulo? codigo $=1070808$

Baumrind, D. (1991). The influence of parenting style on adolescent competence and substance use. The Journal of Early Adolescence, 11(1), 56-95. http://dx.doi.org /10.1177/0272431691111004

Bos, A. E., Muris, P., Mulkens, S., \& Schaalma, H. P. (2006). Changing self-esteem in children and adolescents: A roadmap for future interventions. Netherlands Journal of Psychology, 62 (1), 26-33. http://dx.doi.org/ 10.1007/BF03061048

Cabrera, N. J., Fagan, J., \& Schadler, C. (2012). The influence of mother, father, and child risk on parenting and children's cognitive and social behaviors. Child Development, 82 (6), 1985-2005. http://dx.doi.org/10.111 1/j.1467-8624.2011.01667.x. The

Cardozo, G., \& Alderete, A. M. (2009). Adolescentes en riesgo psicosocial y resiliencia. Psicología Desde El Caribe, 23, 148-182. Recuperado de http://pepsic.bvsa lud.org/pdf/pdc/n23/n23a09.pdf

Chica Olmo, J., \& Frías Jamilena, D. M. (2000). Regresión lineal. En T. L. Martinez (Ed.), Técnicas de análisis de datos en investigaciones de mercados. Madrid, España: Ediciones Pirámide.

Çiftçi, H. D. (2015). Relationship between Aggression and Self-Concept in Nine and Ten-Year- Old Children. Academic Research International, 6(2), 181-198. Retrieved from www.journals.savap.org.pk

Clerici, G., \& García, M. J. (2011). Autoconcepto y pautas de apoyo y control parental en niños escolares. En Memorias del III Congreso Internacional de Investigación y Práctica Profesional en Psicología (pp. 3739). Buenos Aires, Argentina: Secretaría de Investigaciones de la Facultad de Psicología de la Universidad de Buenos Aires.

Cohen, J. (1992). A power primer. Psychological Bulletin, 112(1), 155-159. http://dx.doi.org /10.1037/0033-2909.112.1.155

Coplan, R. J., Findlay, L. C., \& Nelson, L. J. (2004). Characteristics of preschoolers with lower perceived competence. Journal 
of Abnormal Child Psychology, 32(4), 399408. http://dx.doi.org/10.1023/B:JACP.000 0030293.81429 .49

Cross, S. E., \& Madson, L. (1997). Models of the self: Self-construals and gender. Psychological Bulletin, 122(1), 5-37. http://d x.doi.org/10.1037/0033-2909.122.1.5

De La Iglesia, G., Ongarato, P., \& Fernández Liporace, M. (2011). Evaluación de estilos parentales percibidos: Un breve recorrido histórico. En Memorias del III Congreso Internacional de Investigación y Práctica Profesional en Psicología (pp. 40-43). Buenos Aires, Argentina: Secretaría de Investigaciones de la Facultad de Psicología de la Universidad de Buenos Aires.

De la Torre, I. M., Cubillas Rodríguez, J. M., Román Pérez, R., \& Valdez, E. A. (2009). Ideación suicida en población escolarizada infantil: Factores psicológicos asociados. Salud Mental, 32 (6), 495-502. Recuperado de http://www.redalyc.org/pdf/582/582122 67007.pdf

Ferrán Aranaz, M. (1996). SPSS para Windows. Programación y análisis estadístico. Madrid, España: Mc Graw Hill.

Frank, G., Plunkett, S. W., \& Otten, M. P. (2010). Perceived parenting, self-esteem, and general self-efficacy of iranian american adolescents. Journal of Child and Family Studies, 19(6), 738-746. http://dx.doi.org/1 0.1007/s10826-010-9363-x

Fuentes, M. C., García, F., Gracia, E., \& Alarcón, A. (2014). Los estilos parentales de socialización y el ajuste psicológico. Un estudio con adolescentes españoles. Revista de Psicodidactica, 20(1), 117-138. http://dx. doi.org/10.1387/RevPsicodidact.10876

Gorostegui, M.E., \& Dörr, A. (2005). Género y autoconceptos: Un análisis comparativo de las diferencias por sexo en una muestra de niños de educación general básica (EGB) (1992-2003). Psykhe, 14(1), 151-163. Recuperado de http://www.scielo.cl/scielo.php?pid=SO $718-22282005000100012 \&$ script $=$ sci_artt ext
Hair, J., Anderson, R., Tatham, R., \& Black, W. (2001). Análisis multivariante (5th ed.). Madrid: Prentice Hall Iberia.

Harter, S. (1985). Manual for the Self-Perception Profile for Children. Denver, CO: University of Denver.

Harter, S. (1999). The Construction of the Self: A Developmental Perspective (1st ed.). New York, NY: The Guilford Press.

Harter, S., \& Bukowski, W. M. (2012). The construction of the self: Developmental and sociocultural foundations (2nd ed.). New York, NY: The Guilford Press.

Hernández Sampieri, R., Fernández-Collado, C., \& Baptista Lucio, P. (2010). Metodología de la investigación [Research methodology] (5ta ed.). México: Mc Graw Hill.

Ivanova, M. Y., \& Israel, A. C. (2006). Family stability as a protective factor against psychopathology for urban children receiving psychological services. Journal of Clinical Child and Adolescent Psychology, 35 (4), 564-70. http://dx.doi.org/10.1207/s 15374424jccp3504_7

Jiang, Y., Song, J., Lee, M., \& Bong, M. (2014). Self-efficacy and achievement goals as motivational links between perceived contexts and achievement. Educational Psychology, 34(1), 92-117. http://dx.doi.org $/ 10.1080 / 01443410.2013 .863831$

Jiménez, T. I., Murgui, S., \& Musitu, G. (2007). Comunicación familiar y ánimo depresivo: El papel mediador de los recursos psicosociales del adolescente. Revista Mexicana de Psicología, 24(2), 259271. Recuperado de http://www.redalyc.org /pdf/2430/243020637010.pdf

Josephs, R. A., Markus, H., \& Romin, W. T. (1992). Gender and selfesteem. Journal of Personality and Social Psychology, 63(3), 991-402. http:// dx.doi.org/10.1037/0022-3514.63.3.391

Lamb, M. E., \& Tamis-Lemonda, C. S. (2004). The role of the father. An introduction. In M. E. Lamb (Ed.), The rol of the father in child development (4th ed., pp. 1-31). Hoboken, NJ: John Wiley \& Sons. 
Mann, M., Hosman, C. M., Schaalman, H. P., $\&$ de Vries, N. K. (2004). Self-esteem in a broad-spectrum approach for mental health promotion. Health Educational Research. Theory EB Practice, 19(4), 357-372. http:// dx.doi.org/10.1093/her/cyg041

Martínez, I., García, J. F., \& Yubero, S. (2007). Parenting styles and adolescents' self-esteem in Brazil. Psychological Reports, 100 (3), 731-745. http://dx.doi.org/10.2466 /PR0.100.3.731-745

Minichiello, C., Maglio, A. L., \& Schmidt, V. (2010). La familia: Importancia y vigencia de sus funciones. En N. B. Leibovich \& V. Schmidt (Eds.), Ecoevaluación psicológica del contexto familiar. Aspectos teóricos y empíricos (pp. 27-32). Buenos Aires, Argentina: Editorial Guadalupe.

Miñano, P., Gilar, R., \& Castejón, J. L. (2012). Un modelo estructural de variables cognitivo-motivacionales explicativas del rendimiento académico en Lengua Española y Matemáticas. Anales de Psicología, 28(1), 45-54

Molina, M. F., \& Raimundi, M. J. (2011). Predictores de la autoestima global en niños de escuela primaria de la Ciudad de Buenos Aires. Diferencias en función del sexo y la edad. Revista Argentina de Ciencias Del Comportamiento, 3(3), 1-7. Retrieved from http://www.revistas.unc.edu.ar/index. php/racc/article/view/5201

Molina, M. F., Raimundi, M. J., López, C., Cataldi, S. \& Bugallo, L. (2011). Adaptación del Perfil de Autopercepciones para Niños para su Uso en la Ciudad de Buenos Aires. Revista Iberoamericana de Diagnóstico y Evaluación Psicológica (RIDEP), 32(2), 53-78.

Muñoz Silva, A. (2005). La familia como contexto de desarrollo infantil. Dimensiones de análisis relevantes para la intervención educativa y social [Family as context of child development. Main components to educational and social intervention]. Portularia, 5(2), 147-163. Recuperado de http://rabida.uhu.es/dspace/bitstream/h andle/10272/505/b1518923.pdf?sequence $=1$

Oliva Delgado, A., Parra Jiménez, Á., \& Arranz, E. (2008). Estilos relacionales parentales y ajuste adolescente. Infancia Y Aprendizaje, 31 (1), 93-106. http://doi.org/http://dx.doi. org/10.1174/021037008783487093

Oyserman, D., \& Fryberg, S. (2006). The possible selves of diverse adolescents: Content and function across gender, race and national origin. In C. Dunkel \& J. Kerpelman (Eds.), Possible selves: Theory, research, and applications (pp. 1-23). New York: Nova Science Publishers.

Pardo, A., \& Ruiz, M. A. (2005). Análisis de datos con SPSS 13 Base. Madrid, España: McGraw Hill.

Ramos-Díaz, E., Rodríguez-Fernández, A., Fernández-Zabala, A., Revuelta, L., \& Zuazagoitia, A. (2016). Apoyo social percibido, autoconcepto e implicación escolar de estudiantes adolescentes. Revista de Psicodidáctica, 21 (2), 339-356. http://dx. doi.org/10.1387/RevPsicodidact.14848

Reina, C., Oliva Delgado, A., \& Parra Jiménez, Á. (2010). Percepciones de autoevaluación: Autoestima, autoeficacia y satisfacción vital en la adolescencia [Self-evaluation perceptions: Self-esteem, self-efficacy and life satisfaction in adolescence]. Psychology, Society, Ë Education, 2(1), 47-59.

Resett, S., Meier, L., García Sánchez, O., \& Katz, O. (2016). Predicción del autoconcepto a partir de las emociones maternas en niños de edad escolar y diferencias según sexo. Psicodebate, 16(1), 27-48. http:// dx.doi.org/10.18682/pd.v16i1.565

Richaud de Minzi, M. C. (2006a). Loneliness and depression in middle and late childhood: The relationship to attachment and parental style. The Journal of Genetic Psychology, 167(2), 189-210. http:// dx.doi.org/10.3200/GNTP.167.2.189-210

Richaud de Minzi, M. C. (2006b). Parental styles and attachment in relation with self control, social skills and coping in children at risk due to poverty-related causes. In D. M. Devore (Ed.), New developments 
in parent-child relations (pp. 87-110). New York, NY: Nova Science.

Richaud de Minzi, M. C. (2007). La percepción de estilos de relación con su padre y madre en niños y niñas de 8 a 12 años [Childrens ' perception of parental behavior in 8-12 years-old subjects]. Revista Iberoamericana de Diagnóstico Y Evaluación Psicológica, 1(23), 63-81.

Rodríguez, M. A., del Barrio, M. V., \& Carrasco, M. A. (2009). ¿Cómo perciben los hijos la crianza materna y paterna? Diferencias por edad y sexo. Escritos de Psicología, 2(2), 10 18. Recuperado de http://scielo.isciii.es/pdf /ep/v2n2/original2.pdf

Rodríguez-Fernández, A., Ramos-Díaz, E., Ros, I., Fernández-Zabala, A., \& Revuelta, L. (2016). Bienestar subjetivo en la adolescencia: El papel de la resiliencia, el autoconcepto y el apoyo social percibido. Suma Psicológica, 23(1), 60-69. http://dx.d oi.org/10.1016/j.sumpsi.2016.02.002

Schmidt, V., Messoulam, N., \& Molina, M. F. (2008). Autoconcepto académico en adolescentes de escuelas medias: Presentación de un instrumento para su evaluación. Revista Iberoamericana de Diagnóstico Y Evaluación Psicológica, 25(1), 81-106. Recuperado de http://www.aidep.o rg/03_ridep/R25/R254.pdf

Siffert, A., Schwarz, B., \& Stutz, M. (2012). Marital conflict and early adolescents' self-evaluation: the role of parenting quality and early adolescents' appraisals. Journal of Youth and Adolescence, 41 (6), 749-763. http://dx.doi.org/10.1007/ s10964-011-9703-1

Swann, W. B., \& Bosson, J. K. (2010). Self and identity. In G. L. Susan T. Fiske, Daniel T. Gilbert (Ed.), Handbook of social psychology (5th ed., pp. 589-628). New Jersey: John Wiley \& Sons.

Torío López, S., Peña Calvo, V. J., \& Rodríguez Menéndez, M. C. (2008). Estilos educativos parentales. Revisión bibliográfica y reformulación teórica [Parenting styles. Bibliographical revision and theoretical reformulation].
Teoría de La Educación. Revista Interuniversitaria, 20, 151-178. Recuperado de http://gredos.usal.es/jspui/bitstream/103 66/71805/1/Estilos_educativos_parentales _revision_b.pdf

Vargas Rubilar, J. A., \& Oros, L. B. (2013). Parentalidad y autoestima de los hijos: una revisión sobre la importancia del fortalecimiento familiar para el desarrollo infantil positivo. Apuntes Universitarios, 1(1), 155-171. http://dx.doi.org/10.17162/ au.v0i1.13

Villalobos, J. A., Cruz, A. V., \& Sánchez, P. R. (2004). Estilos parentales y desarrollo psicosocial en estudiantes de Bachillerato [Parental styles and psychosocial development in high school students]. Revista Mexicana de Psicología, 21, 119-129.

Wu, P.-C., \& Kuo, S.-T. (2014). Academic achievement, self-concept and depression in Taiwanese children: Moderated mediation effect. School Psychology International, 36(1), 36-53. http://dx.doi.or $\mathrm{g} / 10.1177 / 0143034314559869$

\section{Notas}

* Artículo de investigación 